Supplement of Geosci. Model Dev., 11, 3089-3108, 2018

https://doi.org/10.5194/gmd-11-3089-2018-supplement

(C) Author(s) 2018. This work is distributed under

the Creative Commons Attribution 4.0 License.

(c) (1)

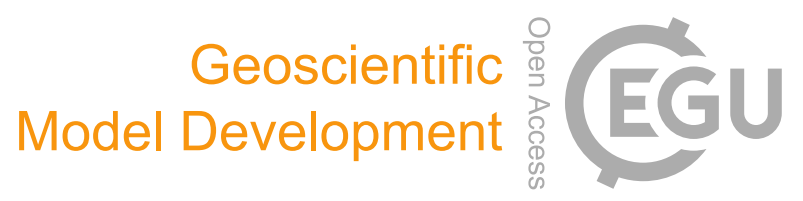

Supplement of

\title{
Quasi-Newton methods for atmospheric chemistry simulations: implementation in UKCA UM vn10.8
}

Emre Esentürk et al.

Correspondence to: Emre Esentürk (e.esenturk.1@warwick.ac.uk)

The copyright of individual parts of the supplement might differ from the CC BY 4.0 License. 


\section{Extended UKCA Box-Model analysis.}

The following is a short discussion of the extra model scenarios carried out for the BOX-MODEL analysis: Rural, Marin and Strat. Equivalent figures to Figures 2 and 3 from the main paper are included for each of these scenarios. The Urban scenario proved to be the most challenging to solve, and have the largest errors, so is shown in the main paper. The QN methods proved to be robust and improved solving speed in all scenarios. However, the choice of what was the ideal iteration number to call the QN iterations varied from scenario to scenario. In the Urban scenario, calling the QN iteration after the first NR iteration often increased the number of iterations required to reach a stable solution, so was counter-productive. In the Rural, Marine and Strat runs, calling the QN method on the first iteration is generally effective at reducing the number of NR iterations required. However, given that the Urban cases were generally the most challenging to solve, the risk of calling the QN on the first iteration causing increase instability was considered to be great. In all scenarios, the QN2-3 experiment consistently improved over the CTNL run, so was the setup chosen to be used in the 3D model.
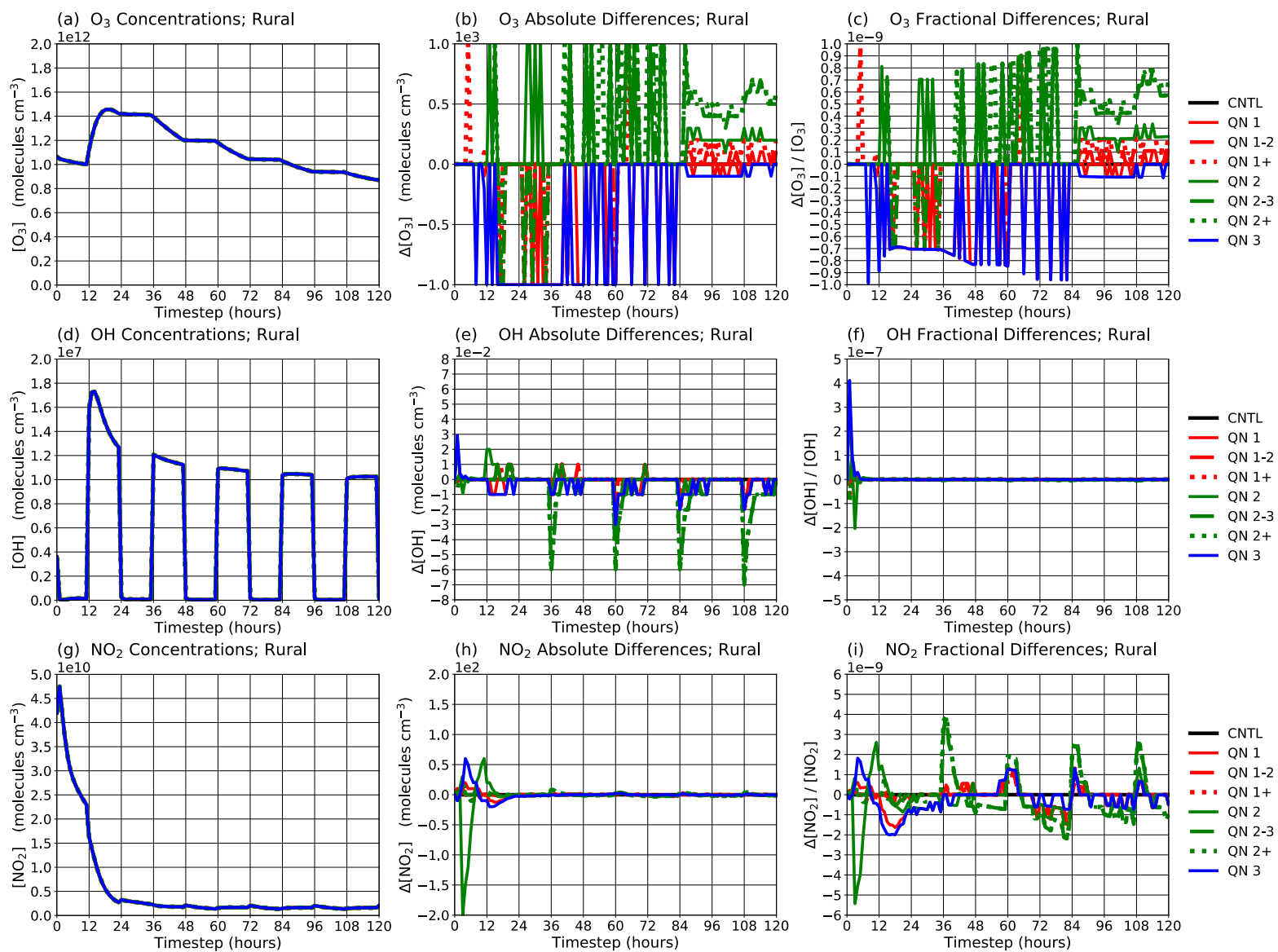

Figure S1. Same as Figure 2 in main paper, but for the Rural scenario 
(a) Rural, CNTL

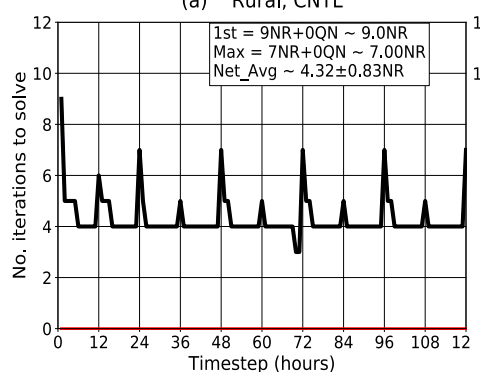

(d) Rural, QN 1-3

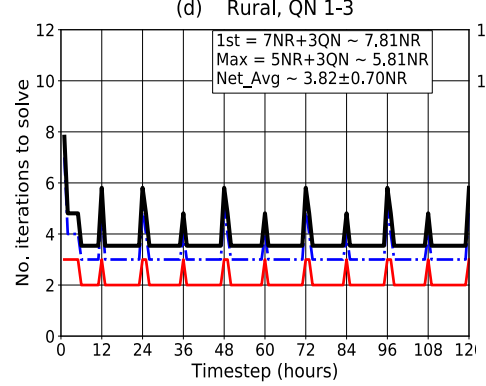

(g) Rural, QN 2-3

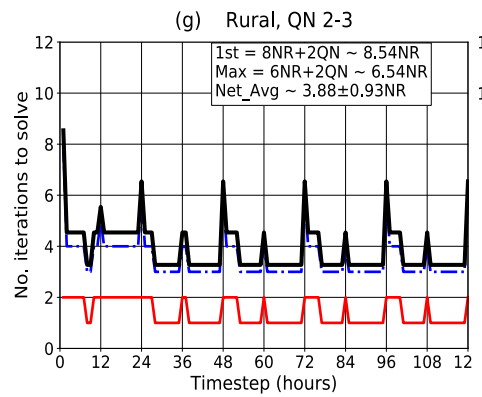

(b) Rural, QN 1

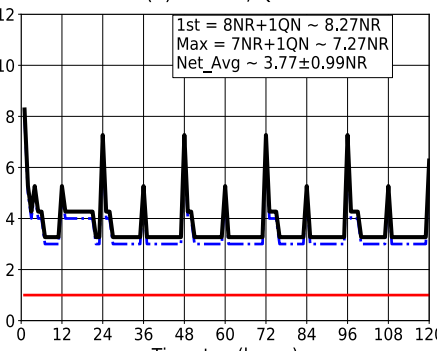

(e) Rural, QN 1+

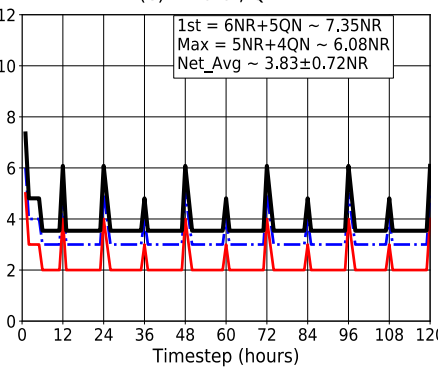

(h) Rural, QN 2+

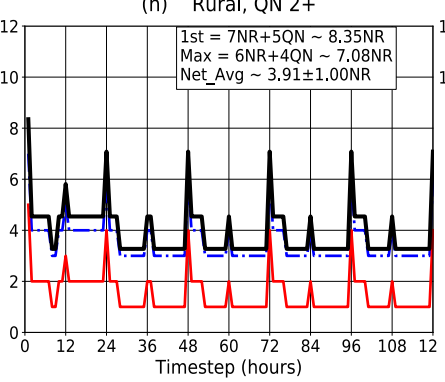

(c) Rural, QN 1-2

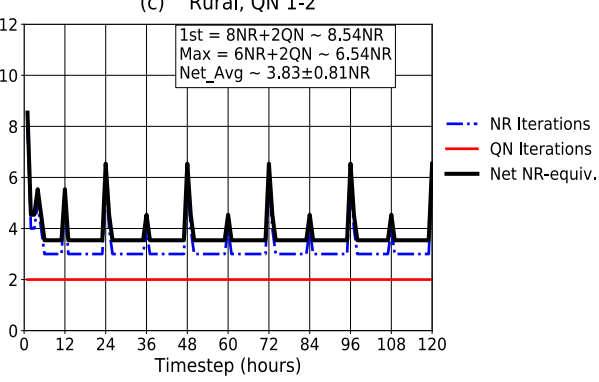

(f) Rural, QN 2

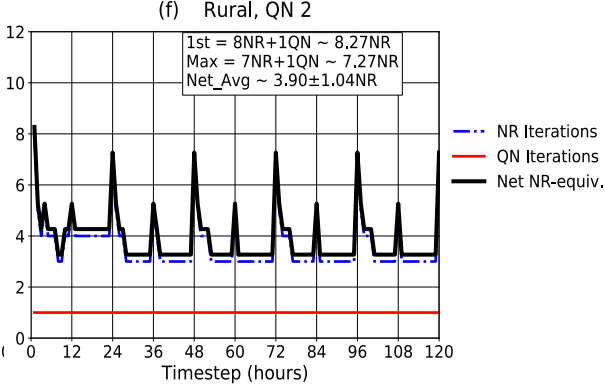

(i) Rural, QN 3

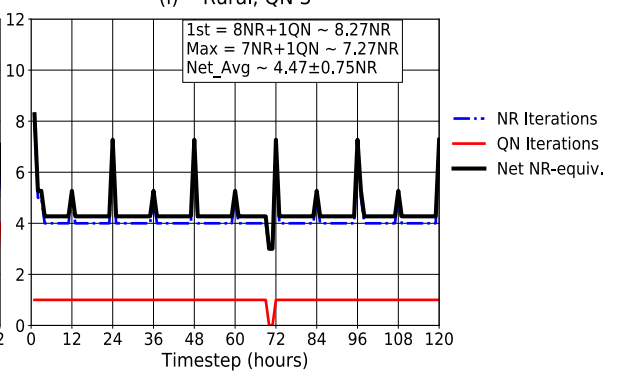

Figure S2. Same as Figure 3 in main paper, but for the Rural scenario 


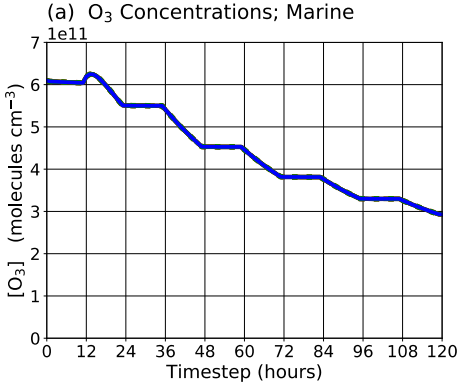

(d) $\mathrm{OH}$ Concentrations; Marine

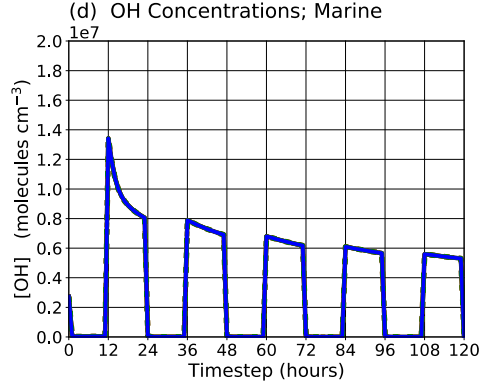

(g) $\mathrm{NO}_{2}$ Concentrations; Marine

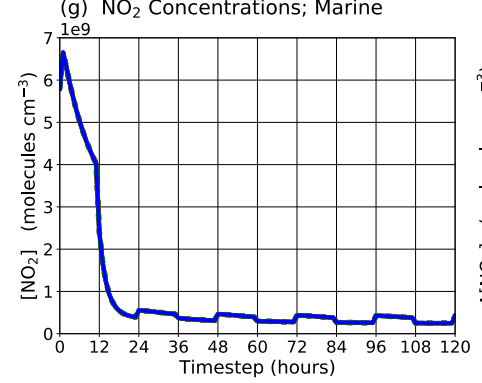

(b) $\mathrm{O}_{3}$ Absolute Differences; Marine

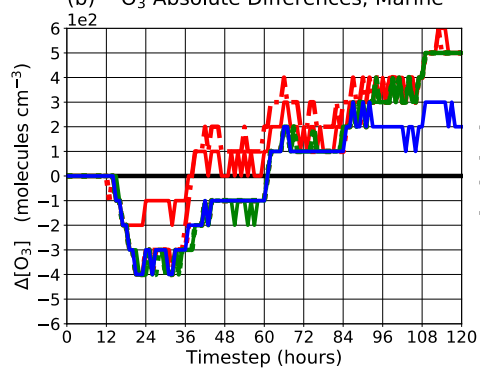

(e) $\mathrm{OH}$ Absolute Differences; Marine

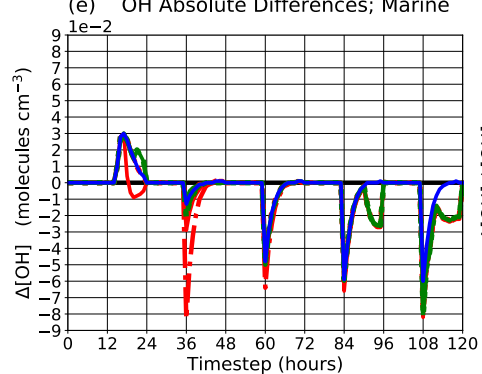

(h) $\mathrm{NO}_{2}$ Absolute Differences; Marine

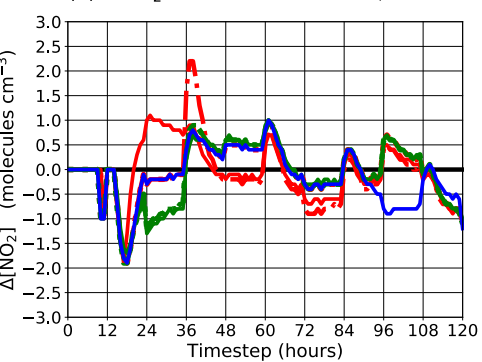

(c) $\mathrm{O}_{3}$ Fractional Differences; Marine
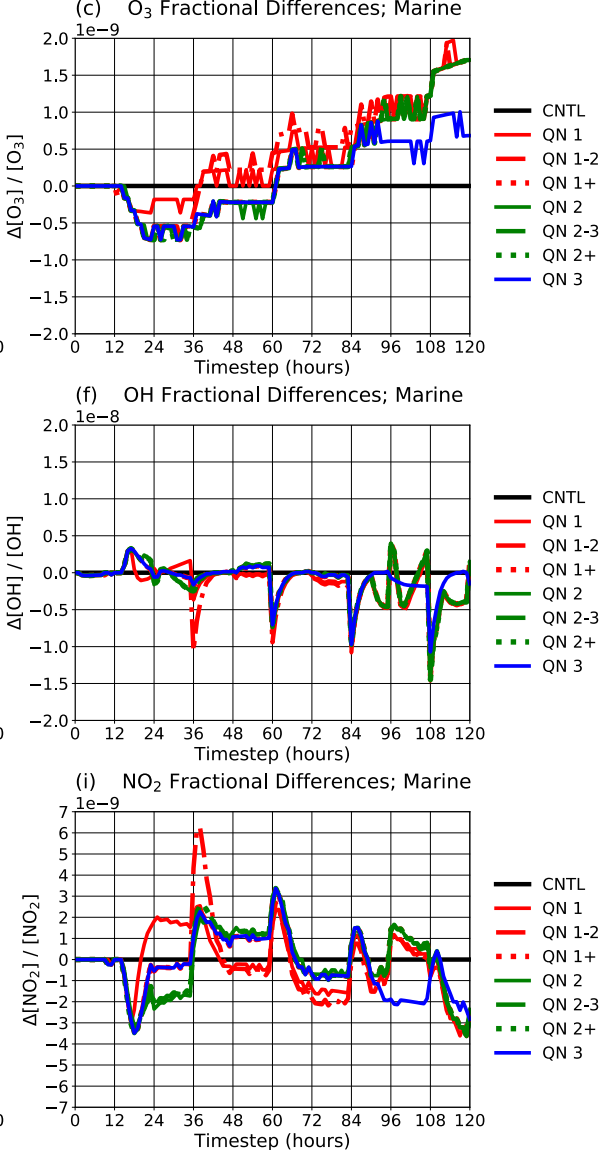

Figure S3. Same as Figure 2 in main paper, but for the Marine scenario 
(a) Marine, CNTL

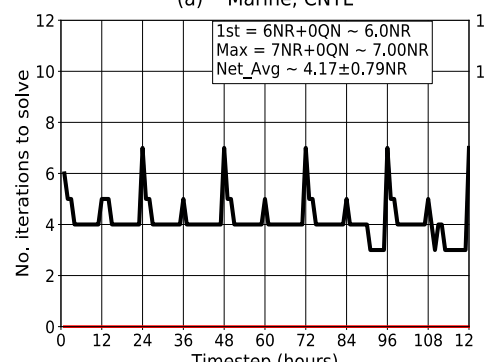

(d) Marine, QN 1-3

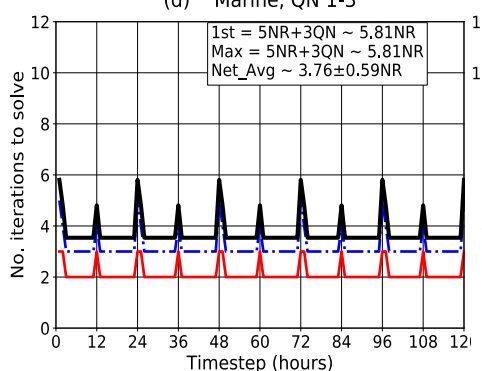

(g) Marine, QN 2-3

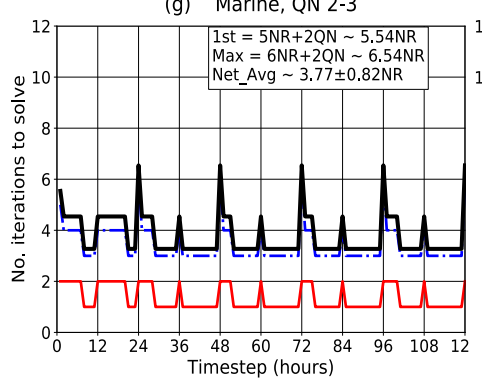

(b) Marine, QN 1

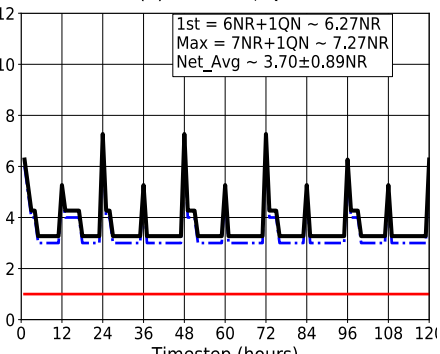

(e) Marine, QN 1+

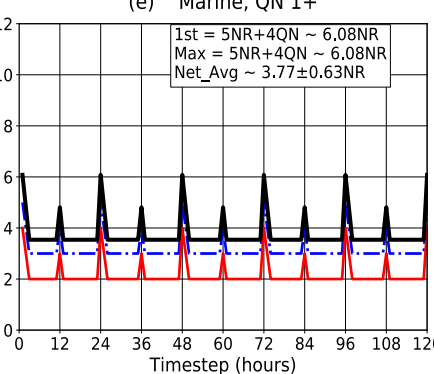

(h) Marine, QN 2+

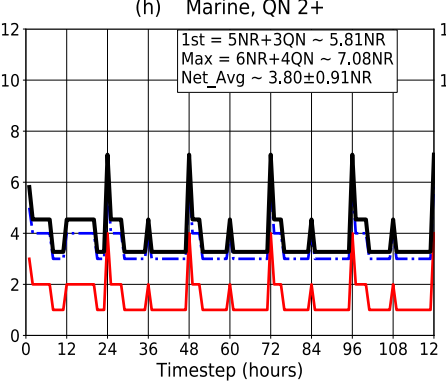

$\begin{array}{lllllll}0 & 12 & 24 & 36 & 48 & 60 & 72 \\ & & & & \\ \text { Timestep (hours) }\end{array}$

(c) Marine, QN 1-2

(f) Marine, QN 2

(i) Marine, QN 3
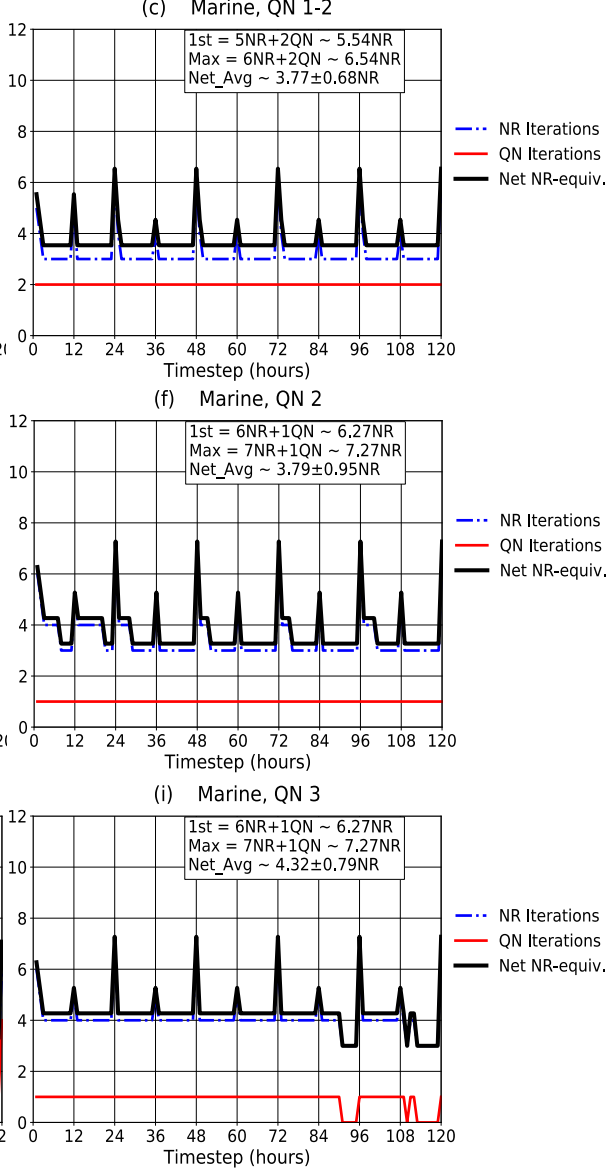

Figure S4. Same as Figure 3 in main paper, but for the Marine scenario 

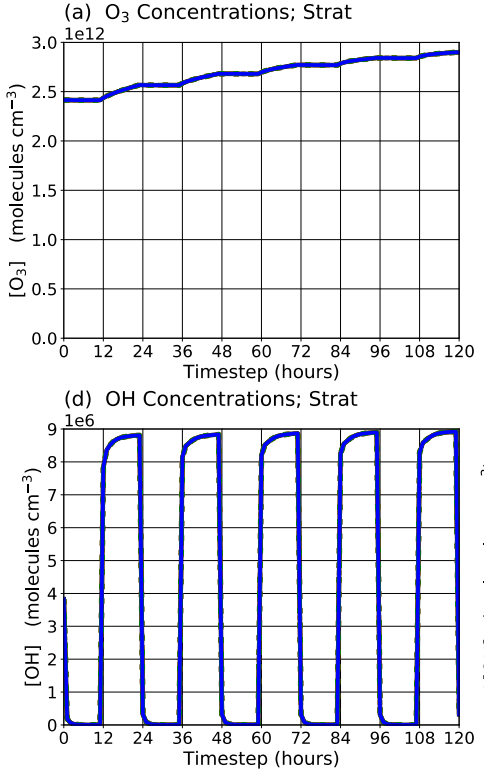

(g) $\mathrm{NO}_{2}$ Concentrations; Strat

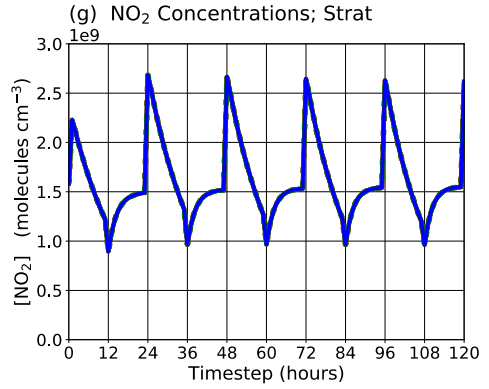

(b) $\mathrm{O}_{3}$ Absolute Differences; Strat

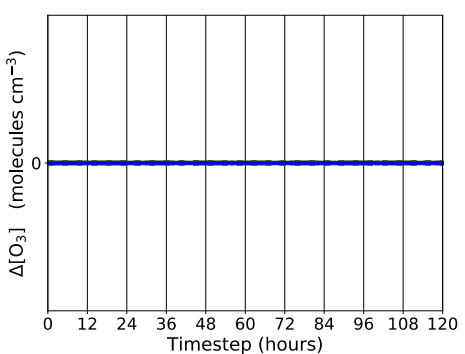

(e) $\mathrm{OH}$ Absolute Differences; Strat

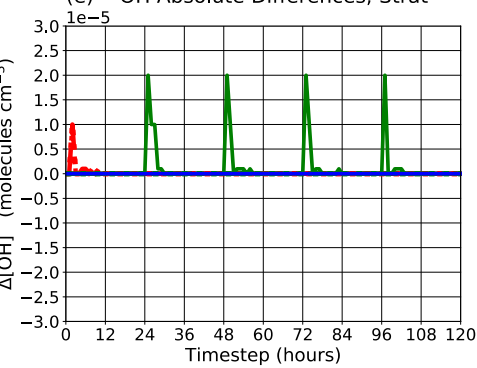

(h) $\mathrm{NO}_{2}$ Absolute Differences; Strat

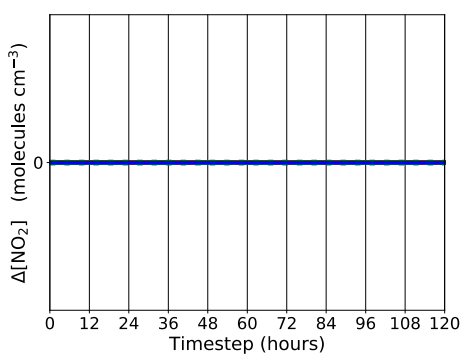

(c) $\mathrm{O}_{3}$ Fractional Differences; Strat
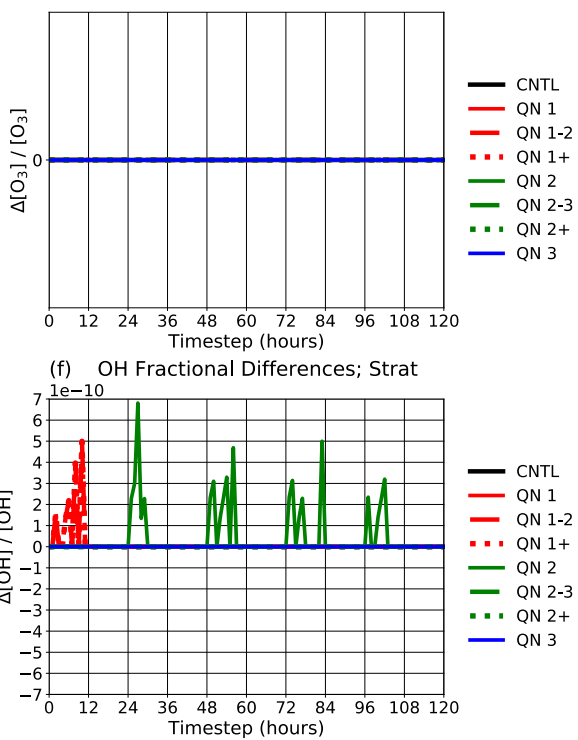

(i) $\mathrm{NO}_{2}$ Fractional Differences; Strat

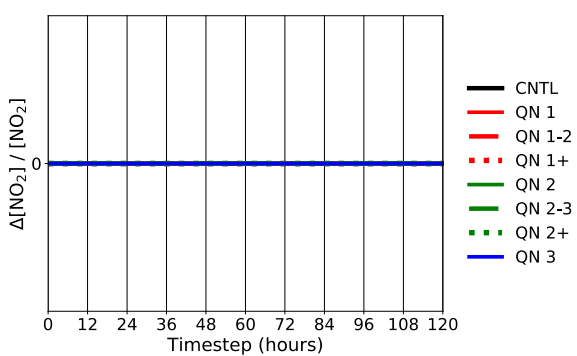

Figure S5. Same as Figure 2 in main paper, but for the Strat scenario 
(a) Strat, CNTL

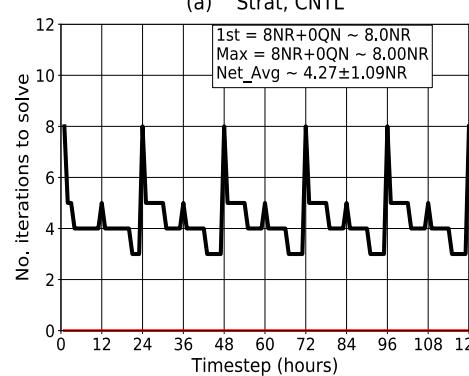

(d) Strat, QN 1-3

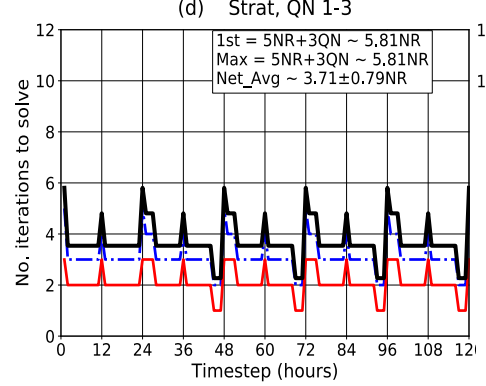

(g) Strat, QN 2-3 (b) Strat, QN 1

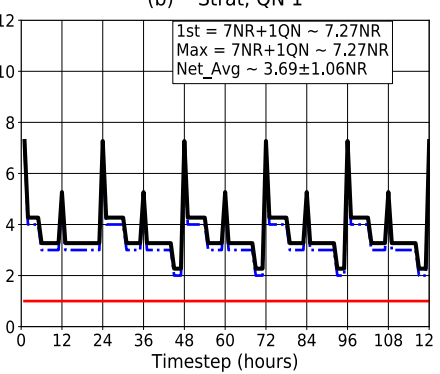

(e) Strat, QN I+

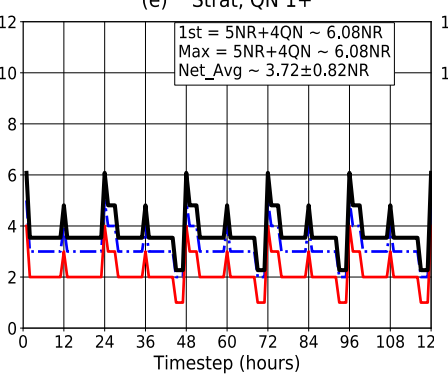

(h) Strat, QN 2+ (c) Strat, QN 1-2

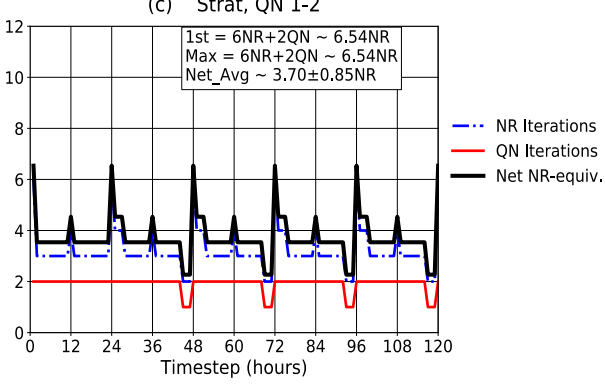

(f) Strat, QN 2

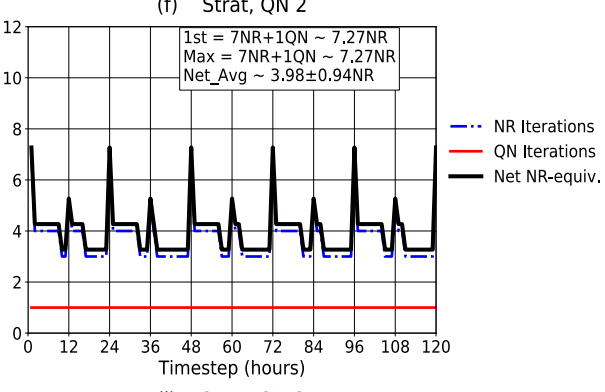

(i) Strat, QN 3

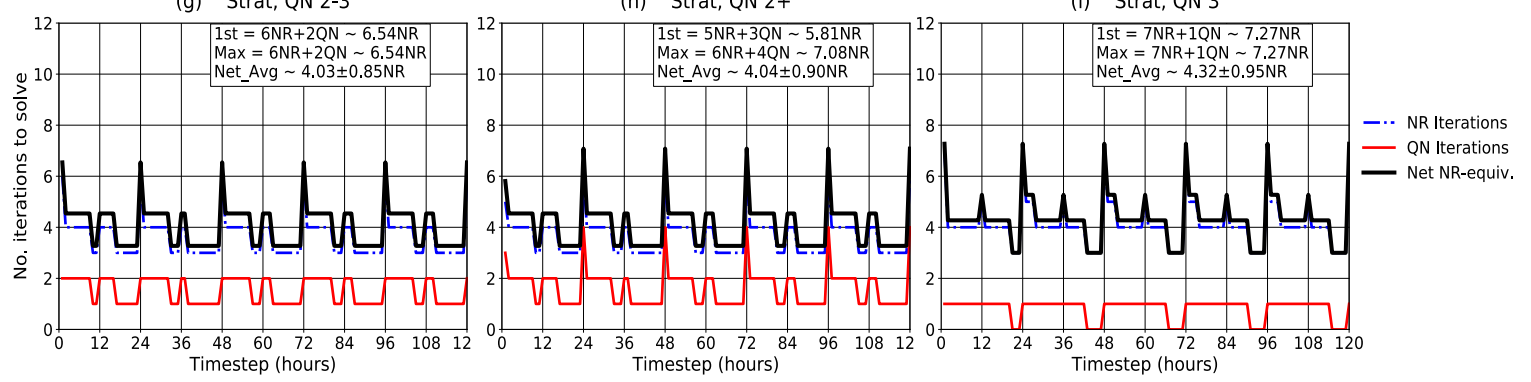

Figure S6. Same as Figure 3 in main paper, but for the Strat scenario

\section{ADDITIONAL UM-UKCA PLOTS and ANALYSIS}

\section{a) QN Performance over 20-year run}

The following plot gives a neat visual of the performance of the QN method for the 20-year run on 432 cores. 


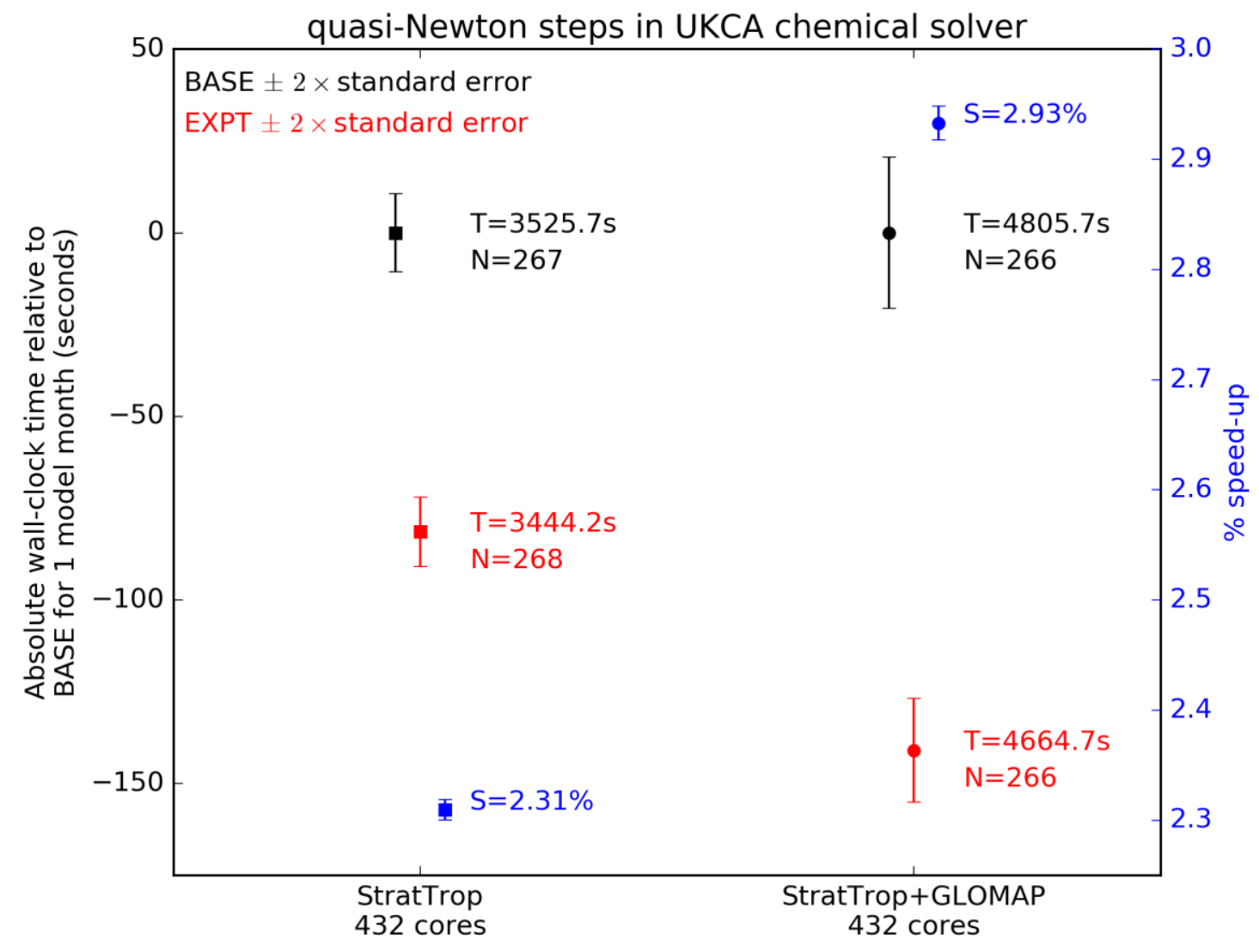

Figure S7. UM speed up for the StratTrop and StratTrop-GLOMAP modes with CNTL and QN implemented runs

\section{b) Time Dependence of Differences Between Simulations and Bias}

For the results to be reliable not only we need to show high accuracy (small difference) as demonstrated in the article but also that the differences between the modified and original simulations do not grow and stay bounded in time.

The first set of three plots (S8-S10) below show, for the OH, normalised absolute mean difference (NMAD), normalised root mean square difference (NRMSD) and normalised mean bias (NMB). The second set of three plots show the same quantities for the Ozone. 


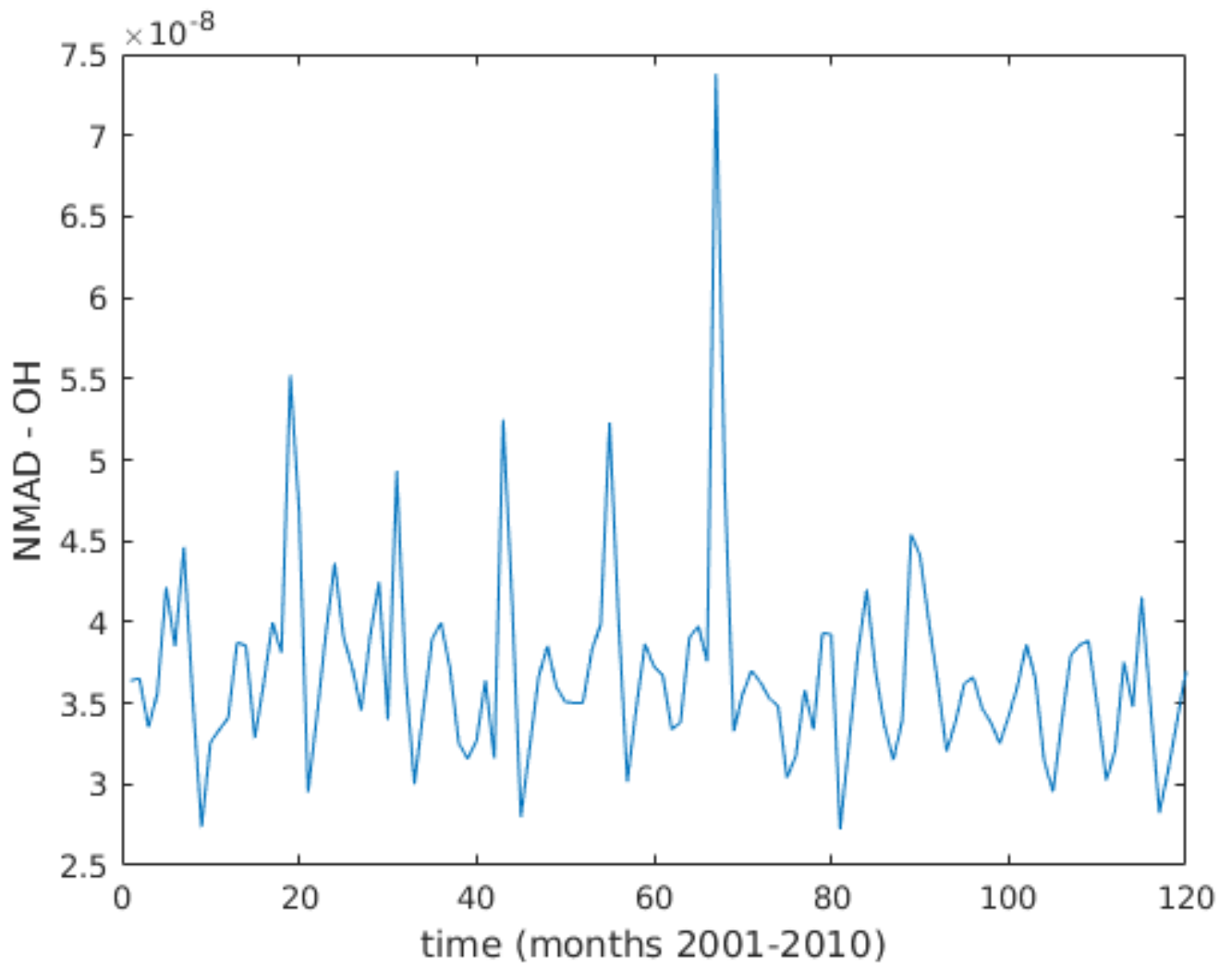

Figure S8. NMAD for monthly averaged OH concentration for the StratTrop Run (Years 2001-2010).

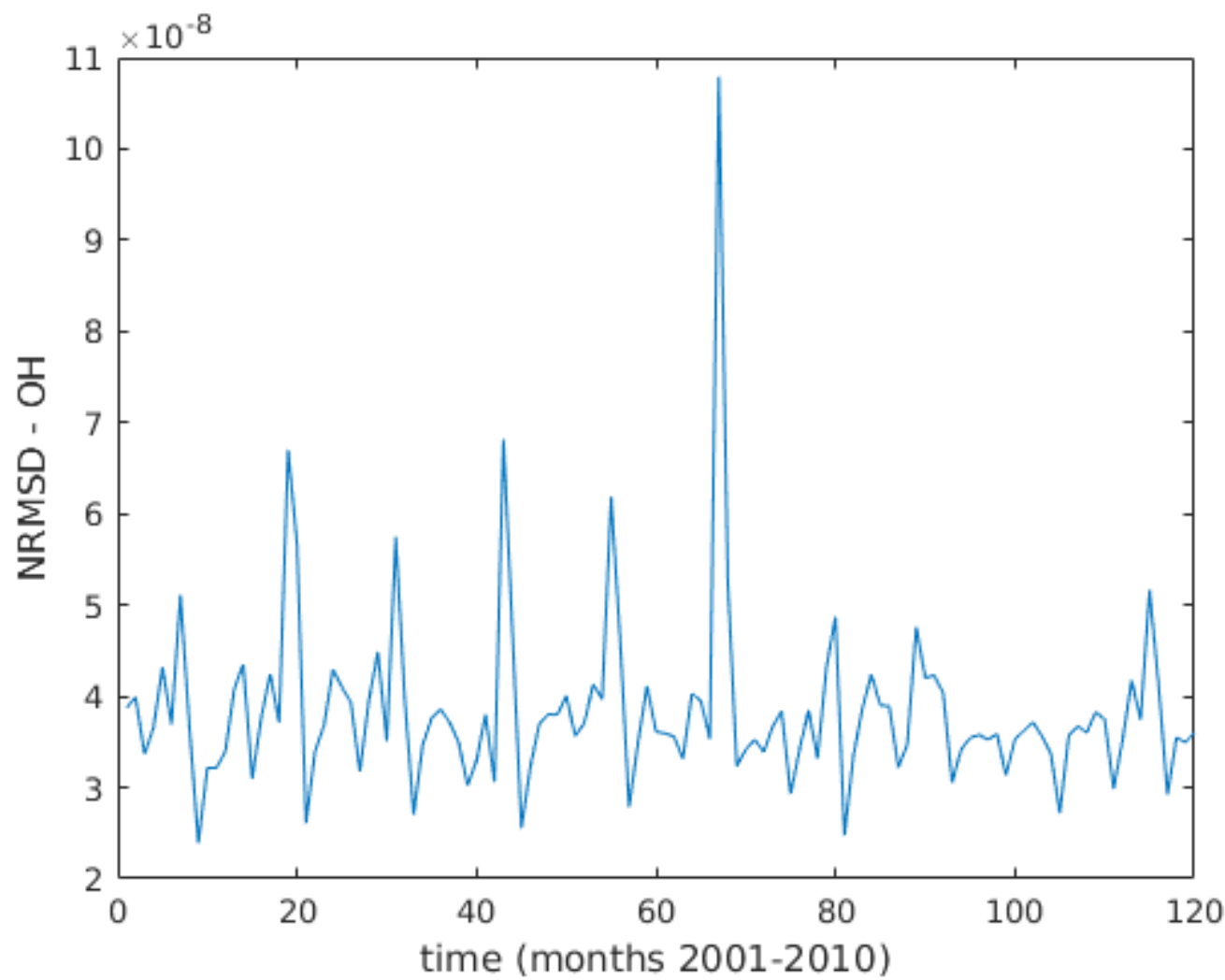

Figure S9. NRMSD for monthly averaged OH concentration for the StratTrop Run (Years 2001-2010). 


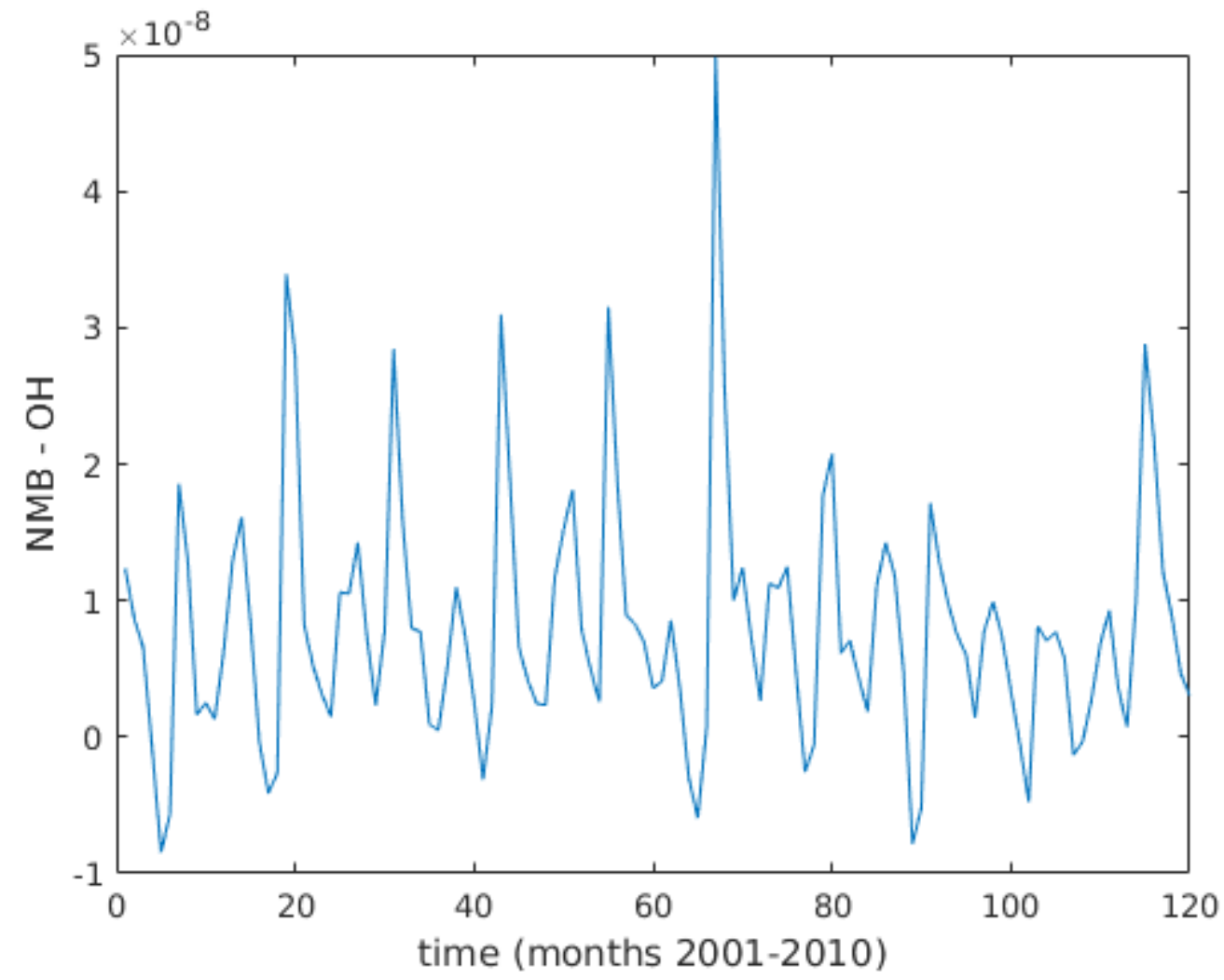

Figure S10. NMB for monthly averaged OH concentration for the StratTrop Run (Years 2001-2010).

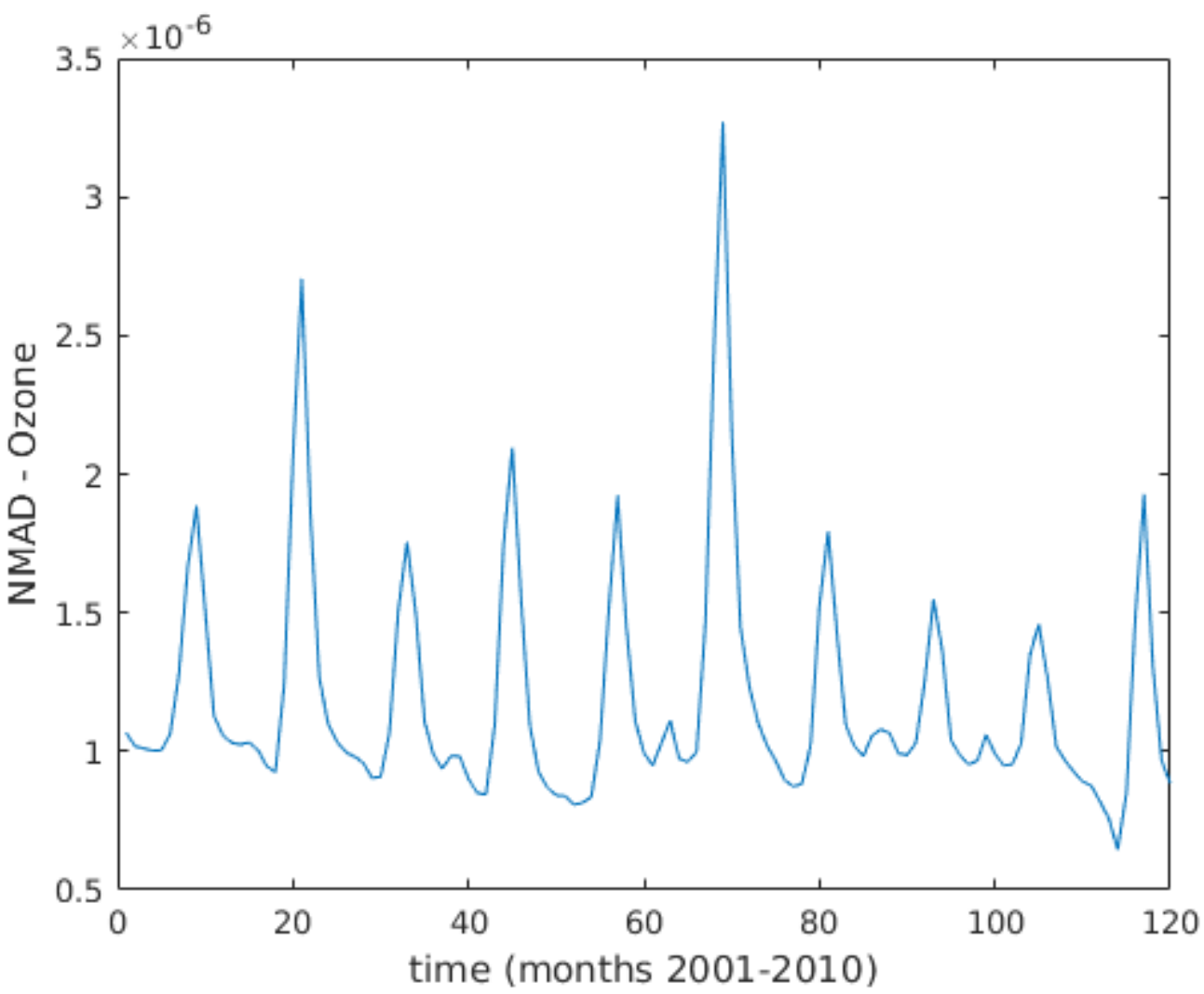

Figure S11. NMAD of monthly averaged Ozone concentration for the StratTrop Run (Years 2001-2010). 


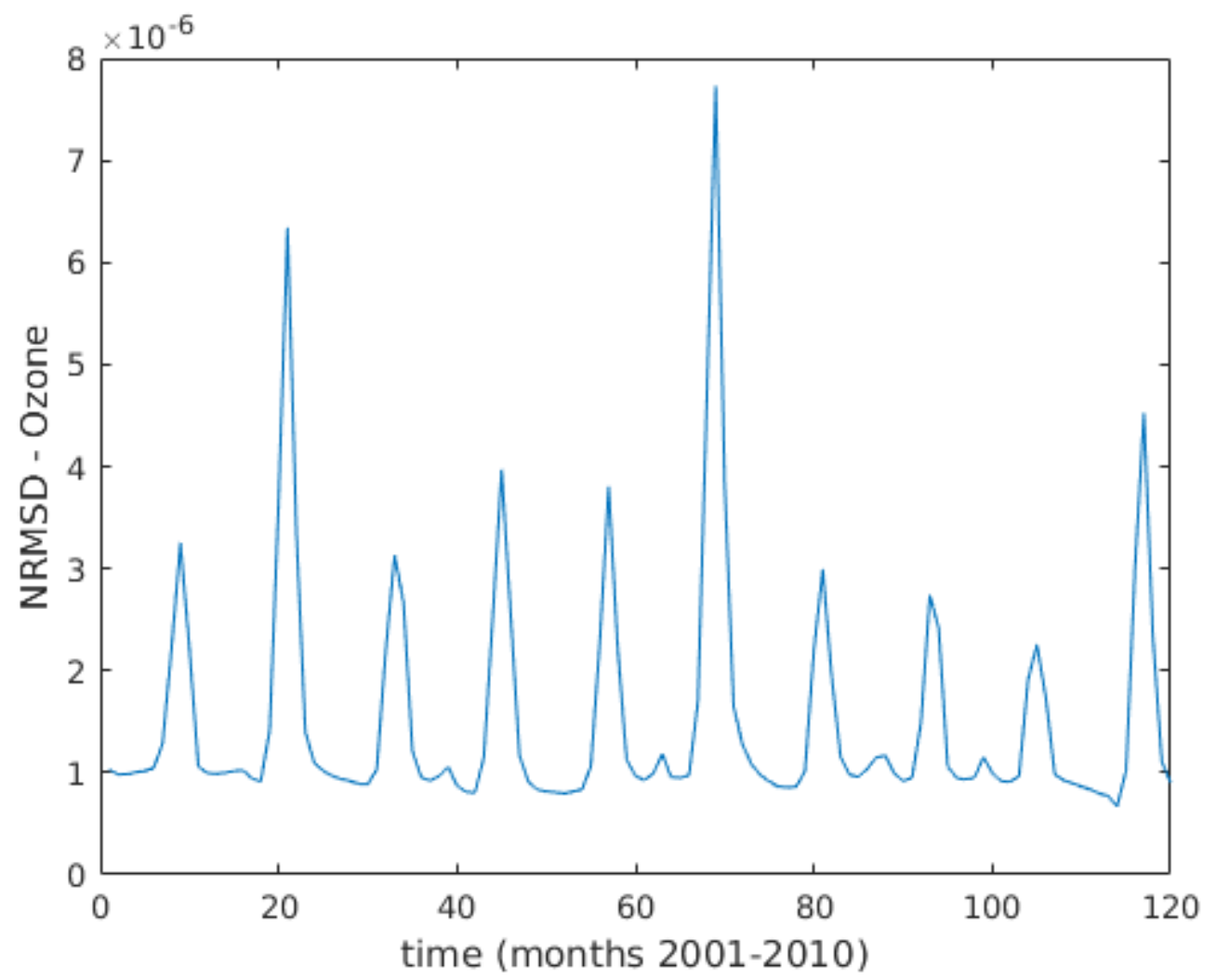

Figure S12. NRMSD of monthly averaged Ozone concentration for the StratTrop Run (Years 2001-2010).

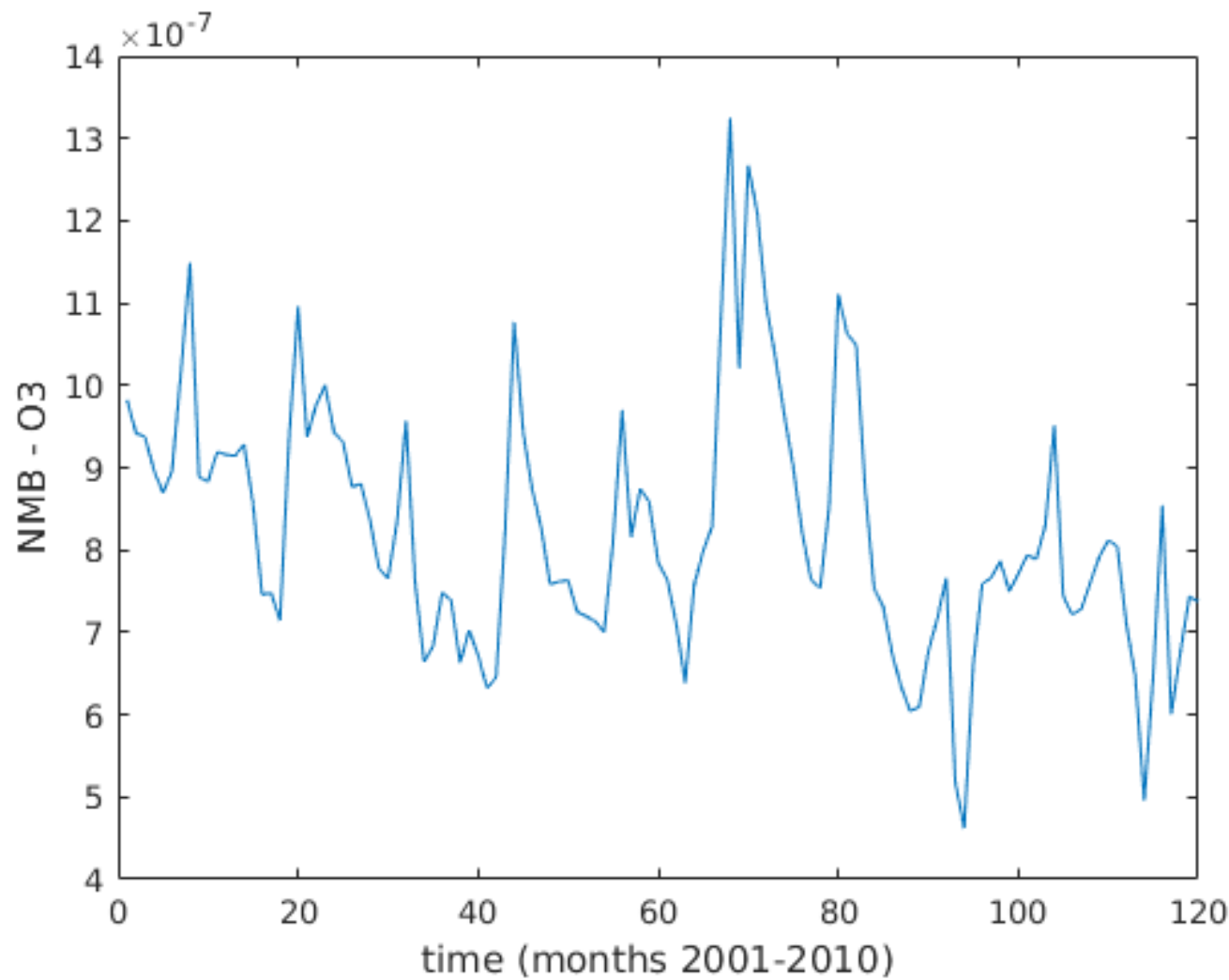

Figure S13. NMB of monthly averaged Ozone concentration for the StratTrop Run (Years 2001-2010). 
c) NMAD, NRMSD and NMB Values for all Species

Below, we give a full table of NMAD, NRMSD and NMB values of all species for the UM-UKCA global model.

\begin{tabular}{|c|c|c|c|c|c|c|}
\hline Species & $\begin{array}{l}\text { NMAD } \\
(* E-3) \\
\text { After } 120 \\
\text { months }\end{array}$ & $\begin{array}{l}\text { NMAD } \\
(* E-3) \\
\text { After } \\
240 \\
\text { months }\end{array}$ & $\begin{array}{l}\text { NRMSD } \\
(* E-3) \\
\text { After } 120 \\
\text { months }\end{array}$ & $\begin{array}{l}\text { NRMSD } \\
(* E-3) \\
\text { After } 240 \\
\text { months }\end{array}$ & $\begin{array}{l}\text { NMB } \\
(* E-3) \\
\text { After } \\
120 \\
\text { months }\end{array}$ & $\begin{array}{l}\text { NMB } \\
(* E-3) \\
\text { After } \\
240 \\
\text { months }\end{array}$ \\
\hline O3 & 0.0011 & 0.00088 & 0.0010 & 0.00090 & 0.00098 & 0.00074 \\
\hline $\mathrm{NO}$ & 0.0441 & 0.0340 & 0.0066 & 0.0077 & -0.0032 & -0.0020 \\
\hline NO3 & 0.0027 & 0.0040 & 0.0074 & 0.0170 & -0.0011 & -0.0021 \\
\hline $\mathrm{N} 2 \mathrm{O} 5$ & 0.0045 & 0.0027 & 0.0052 & 0.0074 & -0.0037 & -0.0016 \\
\hline $\mathrm{HO} 2 \mathrm{NO} 2$ & 0.0053 & 0.0059 & 0.0079 & 0.0086 & -0.0013 & -0.0015 \\
\hline HNO3 & 0.0066 & 0.0058 & 0.0074 & 0.0116 & -0.0046 & -0.0025 \\
\hline $\mathrm{H} 2 \mathrm{O} 2$ & 0.0150 & 0.0172 & 0.0332 & 0.0423 & -0.0135 & -0.0158 \\
\hline $\mathrm{CH} 4$ & 0.0003 & 0.0003 & 0.0004 & 0.0004 & 0.0003 & 0.0003 \\
\hline $\mathrm{CO}$ & 0.0024 & 0.0025 & 0.0019 & 0.0020 & 0.0017 & 0.0017 \\
\hline $\mathrm{HCHO}$ & 0.0089 & 0.0101 & 0.0276 & 0.0343 & -0.0033 & -0.0050 \\
\hline $\mathrm{CH} 3 \mathrm{OOH}$ & 0.0089 & 0.0105 & 0.0180 & 0.0220 & -0.0049 & -0.0064 \\
\hline HONO & 0.0206 & 0.0204 & 0.1723 & 0.2035 & 0.0017 & 0.0040 \\
\hline $\mathrm{C} 2 \mathrm{H} 6$ & 0.0118 & 0.0137 & 0.0098 & 0.0129 & 0.0118 & 0.0136 \\
\hline $\mathrm{C} 2 \mathrm{H} 5 \mathrm{OOH}$ & 0.0156 & 0.1939 & 0.0186 & 0.0279 & 0.0085 & 0.0111 \\
\hline $\mathrm{CH} 3 \mathrm{CHO}$ & 0.0098 & 0.0012 & 0.0010 & 0.0013 & 0.0056 & 0.0067 \\
\hline PAN & 0.0115 & 0.0153 & 0.0319 & 0.0530 & -0.0011 & -0.0006 \\
\hline $\mathrm{C} 3 \mathrm{H} 8$ & 0.0092 & 0.0127 & 0.0071 & 0.0140 & 0.0089 & 0.0123 \\
\hline $\mathrm{n}-\mathrm{C} 3 \mathrm{H} 7 \mathrm{OOH}$ & 0.0140 & 0.0225 & 0.0189 & 0.0412 & 0.0103 & 0.0180 \\
\hline $\mathrm{i}-\mathrm{C} 3 \mathrm{H} 7 \mathrm{OOH}$ & 0.0152 & 0.0237 & 0.0198 & 0.0412 & 0.0103 & 0.0180 \\
\hline $\mathrm{C} 2 \mathrm{H} 5 \mathrm{CHO}$ & 0.0093 & 0.0134 & 0.0408 & 0.0373 & 0.0072 & 0.0101 \\
\hline $\mathrm{C} 2 \mathrm{H} 6 \mathrm{CO}$ & 0.00933 & 0.01099 & 0.00783 & 0.00959 & 0.00926 & 0.01086 \\
\hline $\mathrm{CH} 3 \mathrm{COCH} 2 \mathrm{OOH}$ & 0.01394 & 0.01660 & 0.01950 & 0.02661 & 0.00301 & 0.00494 \\
\hline PPAN & 0.01001 & 0.01193 & 0.03702 & 0.06292 & -0.00931 & -0.01031 \\
\hline
\end{tabular}




\begin{tabular}{|c|c|c|c|c|c|c|}
\hline $\mathrm{CH} 3 \mathrm{ONO} 2$ & 0.01053 & 0.01355 & 0.01481 & 0.01974 & -0.00932 & -0.01286 \\
\hline $\mathrm{C} 5 \mathrm{H} 8$ & 0.02416 & 0.02421 & 0.02180 & 0.02464 & 0.01083 & 0.01182 \\
\hline ISOOH & 0.02798 & 0.02933 & 0.03170 & 0.03713 & 0.01167 & 0.01110 \\
\hline ISON & 0.03209 & 0.04159 & 0.06750 & 0.10933 & 0.00375 & 0.01014 \\
\hline MACR & 0.02345 & 0.02557 & 0.02783 & 0.03507 & 0.01884 & 0.01998 \\
\hline MACROOH & 0.04468 & 0.05189 & 0.05149 & 0.07872 & 0.02563 & 0.03051 \\
\hline MPAN & 0.03073 & 0.03666 & 0.04900 & 0.06991 & -0.00697 & -0.00842 \\
\hline HACET & 0.02630 & 0.03193 & 0.03694 & 0.05458 & -0.00538 & -0.00461 \\
\hline MGLY & 0.14927 & 0.18045 & 0.41070 & 0.63219 & -0.14196 & -0.17186 \\
\hline NALD & 0.04737 & 0.06276 & 0.10608 & 0.17306 & 0.02292 & 0.04034 \\
\hline $\mathrm{HCOOH}$ & 0.01836 & 0.02537 & 0.02768 & 0.04264 & -0.00854 & -0.00811 \\
\hline $\mathrm{CH} 3 \mathrm{CO} 3 \mathrm{H}$ & 0.02581 & 0.03148 & 0.03700 & 0.05843 & 0.01038 & 0.01493 \\
\hline $\mathrm{CH} 3 \mathrm{CO} 2 \mathrm{H}$ & 0.02374 & 0.02903 & 0.03652 & 0.05757 & -0.00118 & 0.00231 \\
\hline MVKOOH & 0.02997 & 0.03284 & 0.05591 & 0.06614 & 0.00713 & 0.00526 \\
\hline $\mathrm{Cl}$ & 0.00069 & 0.00035 & 0.00090 & 0.00036 & -0.00069 & -0.00020 \\
\hline $\mathrm{ClO}$ & 0.00245 & 0.00247 & 0.00738 & 0.00731 & 0.00015 & 0.00064 \\
\hline $\mathrm{Cl} 2 \mathrm{O} 2$ & 0.09651 & 0.05123 & 0.28837 & 0.05308 & 0.00543 & 0.02153 \\
\hline $\mathrm{OClO}$ & 0.01277 & 0.01082 & 0.01654 & 0.01336 & 0.00894 & 0.00849 \\
\hline $\mathrm{Br}$ & 0.00051 & 0.00056 & 0.00048 & 0.00065 & -0.00029 & -0.00023 \\
\hline $\mathrm{BrCl}$ & 0.01104 & 0.00941 & 0.01503 & 0.01262 & 0.00771 & 0.00731 \\
\hline BrONO2 & 0.00471 & 0.00439 & 0.00757 & 0.00754 & -0.00234 & -0.00186 \\
\hline $\mathrm{N} 2 \mathrm{O}$ & 0.00004 & 0.00003 & 0.00008 & 0.00005 & 0.00004 & 0.00003 \\
\hline $\mathrm{HOCl}$ & 0.00639 & 0.00587 & 0.01665 & 0.01657 & 0.00087 & 0.00045 \\
\hline $\mathrm{HBr}$ & 0.008348 & 0.00908 & 0.01627 & 0.015961 & -0.00754 & -0.0083 \\
\hline $\mathrm{HOBr}$ & 0.006061 & 0.00595 & 0.011883 & 0.012055 & 0.00258 & 0.00168 \\
\hline $\mathrm{ClONO} 2$ & 0.001965 & 0.00217 & 0.00272 & 0.0031523 & $-8 \mathrm{E}-05$ & 0.00043 \\
\hline $\mathrm{CFCl3}$ & $4.35 \mathrm{E}-05$ & $3.9 \mathrm{E}-05$ & $8.4 \mathrm{E}-05$ & $7.53 \mathrm{E}-05$ & $4.3 \mathrm{E}-05$ & $3.8 \mathrm{E}-05$ \\
\hline $\mathrm{CF} 2 \mathrm{Cl} 2$ & $3.54 \mathrm{E}-05$ & $2.7 \mathrm{E}-05$ & $7 \mathrm{E}-05$ & $5.03 \mathrm{E}-05$ & $3.3 \mathrm{E}-05$ & $2.5 \mathrm{E}-05$ \\
\hline $\mathrm{CH} 3 \mathrm{Br}$ & 0.00044 & 0.00047 & 0.00052 & 0.00055 & 0.00044 & 0.00047 \\
\hline $\mathrm{N}$ & 0.000798 & 0.00403 & 0.0006 & 0.004242 & -0.00077 & -0.0027 \\
\hline $\mathrm{O}(3 \mathrm{P})$ & $2.66 \mathrm{E}-05$ & $1.3 \mathrm{E}-05$ & $4.6 \mathrm{E}-05$ & $3.19 \mathrm{E}-05$ & $-1.9 \mathrm{E}-05$ & $-5 \mathrm{E}-06$ \\
\hline ORGNIT & 0.056804 & 0.06181 & 0.08377 & 0.107436 & 0.03981 & 0.04092 \\
\hline
\end{tabular}




\begin{tabular}{|l|l|l|l|l|l|l|}
\hline $\mathrm{H}$ & $8.77 \mathrm{E}-06$ & $5.4 \mathrm{E}-06$ & $2.7 \mathrm{E}-05$ & $1.99 \mathrm{E}-05$ & $8.8 \mathrm{E}-06$ & $2.2 \mathrm{E}-06$ \\
\hline $\mathrm{OH}$ & $3.64 \mathrm{E}-05$ & $3.7 \mathrm{E}-05$ & $3.9 \mathrm{E}-05$ & $3.6 \mathrm{E}-05$ & $1.2 \mathrm{E}-05$ & $3 \mathrm{E}-06$ \\
\hline $\mathrm{HO} 2$ & 0.001041 & 0.00108 & 0.00109 & 0.001262 & 0.0003 & 0.00023 \\
\hline $\mathrm{CH} 3 \mathrm{OO}$ & 0.00291 & 0.00492 & 0.00214 & 0.003857 & 0.0025 & 0.00375 \\
\hline $\mathrm{C} 2 \mathrm{H} 5 \mathrm{OO}$ & 0.056128 & 0.0569 & 0.16649 & 0.16476 & 0.04521 & 0.04526 \\
\hline $\mathrm{CH3CO3}$ & 0.051005 & 0.05548 & 0.21106 & 0.274743 & -0.02183 & -0.0224 \\
\hline n-C3H7OO & 0.076912 & 0.08898 & 0.2034 & 0.272403 & 0.07119 & 0.08267 \\
\hline i-C3H7OO & 0.070862 & 0.08225 & 0.19265 & 0.258701 & 0.06177 & 0.07211 \\
\hline $\mathrm{C} 2 \mathrm{H} 5 \mathrm{CO} 3$ & 0.032232 & 0.04066 & 0.11543 & 0.191884 & -0.01635 & -0.0173 \\
\hline $\mathrm{CH} 3 \mathrm{COCH} 2 \mathrm{OO}$ & 0.043401 & 0.04446 & 0.11144 & 0.119976 & 0.01727 & 0.0185 \\
\hline $\mathrm{CH} 3 \mathrm{OH}$ & 0.005674 & 0.00593 & 0.007 & 0.009182 & -0.00167 & -0.003 \\
\hline $\mathrm{HCl}$ & 0.000445 & 0.00049 & 0.00135 & 0.001333 & $-8.3 \mathrm{E}-05$ & -0.0001 \\
\hline $\mathrm{BrO}$ & 0.001422 & 0.00155 & 0.00471 & 0.004844 & -0.00062 & -0.0006 \\
\hline $\mathrm{NO} 2$ & 0.004671 & 0.00346 & 0.00718 & 0.012697 & -0.00346 & -0.002 \\
\hline $\mathrm{O}(1 \mathrm{D})$ & $2.69 \mathrm{E}-05$ & $1.8 \mathrm{E}-05$ & $3.2 \mathrm{E}-05$ & $2.43 \mathrm{E}-05$ & $1.2 \mathrm{E}-05$ & $1.1 \mathrm{E}-05$ \\
\hline
\end{tabular}

